\title{
FONDO EDITORIAL
}

\section{Presentación del Libro “Derrotando}

\section{al Cancer" Del Dr. Carlos Vallejos SOLOGUREN}

Presentation of the Book "Defeating Cancer" by

Dr. Carlos Vallejos Sologuren

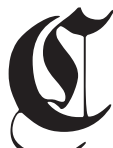
on notable concurrencia de colegas, amistades y familiares, se llevó a cabo la ceremonia de presentación del libro "Derrotando al cáncer. Reseña histórica de la oncología médica en el Perú.

"(1) el pasado jueves 21 de febrero en la Clínica Delgado (Terraza del 4to. piso), Calle Borgoño $\mathrm{s} / \mathrm{n}$ - Miraflores, a las 7:30 pm.

El libro fue comentado por el Dr. Luis Pinilllos Ashton y luego se tuvo las palabras del autor, Dr. Carlos Vallejos Sologuren.

\section{Comentarios Del Dr. Luis Pinillos Ashton}

El cáncer es una enfermedad que ha existido desde tiempos inmemorables pero ha aumentado con el tiempo convirtiéndose en nuestros días en un grave problema de salud pública y en el Perú es la segunda causa de muerte.

La forma de enfrentar esta enfermedad ha evolucionado en el tiempo. Al inicio, en pruebas de ensayo/error y hoy a través de serios estudios de investigación con reglas muy precisas y resultados estimulantes, los

1 Vallejos Sologuren, Carlos. Derrotando al Cáncer. Reseña histórica de la oncología médica en el Perú. Lima: Centro Editorial de la Universidad Peruana Cayetano Heredia; 2019, 257 páginas.

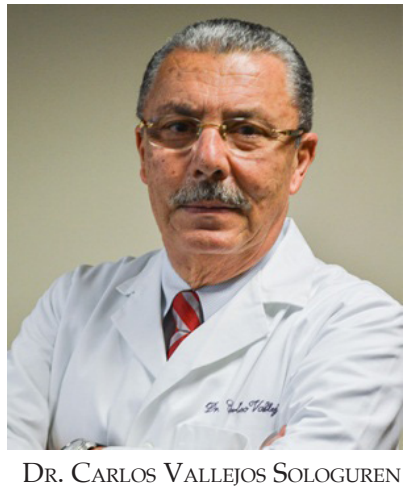

tratamientos han evolucionado tanto en cirugía como radioterapia y quimioterapia.

La quimioterapia ha tenido un desarrollo espectacular a partir de los años 1970 y es por ello que considero nadie puede describir mejor este proceso que Carlos Vallejos Sologuren, que inició su práctica en los años 1970 y sigue liderando la investigación, tratamientos y difusión de conocimientos al día de hoy. Él es ejemplo viviente y actualizado de todos los cambios que conocemos de la especialidad y no solo en el Perú como en América Latina y en el mundo, donde su experiencia es muy reconocida.

Basta decir que ha presidido, FLASCA, SLACOG, la Sociedad Peruana de Cancerología, la de Oncología Médica, lidera ECOG PERÚ, ha sido galardonado como 


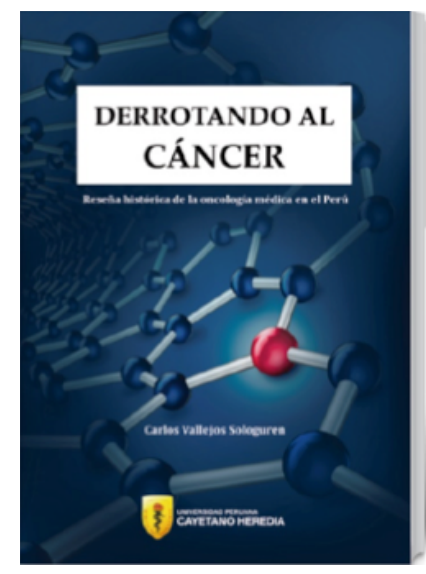

distinguido alumno del MD Anderson Cancer Center, ha sido director del INEN, ministro de salud y podría seguir extendiéndome en su currículum pero hoy se trata de presentar su libro correctamente titulado Derrotando al cáncer. Reseña histórica de la oncología médica en el Perú.

Este libro magníficamente redactado, esencialmente como lectura histórica, es muy fácil de leer con tintes científicos que, se ve, no se aleja nunca de su mente.

Tiene 257 páginas, tres prólogos, fotografías históricas -como la del papiro de los escritos de la Dinastía XVIII de Egipto de los años 1550 antes de Cristo- y definiciones de Hipócrates del karkinos o de Claudio Galeno de onkos, que significa masa, y entre otros presenta contribuciones de Lanfranco de Milan, desterrado a Lyon en 1290 (ruta que curiosamente siguió nuestro amigo Peter Boyle de iPRI).

En sus páginas se describen una serie de hitos en oncología:

- Paracelso, en 1531 describió el cáncer de pulmón.

- Jansen, en 1590, inventó el microscopio.

- El Hospital de Middlesex, Londres, en 1792 se estableció el primer servicio de oncología.

- Percivall Pott, en el siglo XVIII, describió el cáncer de escroto.

- El uyso de la cicuta, muérdago y arsénico en el XVII,I en España.

- Bilroth, lideró la cirugía abdominal, fines del siglo XIX.

- Halsted, realizó una mastectomía en 1882.

- Wertheim, realizó una histerectomia radical en 1898.

- Beatson, llevó a cabo una ooforectomia para el cáncer de mama en 1896.

- Roentgen y los esposos Curie propalaron la radioterapia, a fines del siglo XIX.

- Charles Dodds sintetizó el dietiletilbestrol en 1938.

- E. Freireich. la trasfusión de leucocitos en 1970.

- Charles Huggins, realizó la castración para el tratamiento del cáncer de próstata, en 1940.

- Goodman, empleó la mostaza nitrogenada, en 1944.

- Paul Erlich, Premio Nobel de medicina de 1908 por sintetizar los quimioterápicos.

- Richard Doll, en 1950, describió la relación entre tabaco y cáncer.

El libro presenta homenaje a distintos médicos que impulsaron la oncología con los que el autor ha tenido una estrecha relación profesional y amical: Roberto Esteves, Eduardo Cáceres, Andres Solidoro, E. Freireich, E. Frey, Vincent De Vita, Gianni Bonadona, Umberto Veronesi, Lawrence Einhorn, Bernard Fisher, Robert C. Gallo, Vicente Valero, Hernán Cortez, Esteban Cvitkovic, Luis Ráez, Peter Boyle, Carlos Payet y Luis Pinillos Ganoza.

De ellos aprendió, por ejemplo, lo dicho por Freireich: El médico que sabe tratar leucemia aguda, sabe medicina interna, porque la enfermedad puede afectar cualquier órgano del ser humano.

Una parte muy importante es la referente, 
evidentemente al Perú. Describe que en 1935, el presidente Benavides creó el impuesto al hielo y a las refrigeradoras para que con lo recaudado se monte el primer departamento de ginecología y cáncer en el Hospital Arzobispo Loayza de Lima. El mismo presidente edificó el Instituto de Cáncer, que se inauguró en 1939. Refiere su evolución del Instituto de Cáncer a Instituto de Radioterapia y al Instituto Nacional de Enfermedades Neoplásicas en 1952, y la reorganización de este por el Dr. Eduardo Cáceres, con la creación de departamentos médicos por región anatómica.

Presenta la relación de los primeros residentes a partir de 1952 y entre ellos menciona a los Dres. Andrés Solidoro, Teresa Pasco, Ernesto Salem, Mayer Zaharia, Leonardo Adachi, Fernando Delgado, entre otros.

Presenta también el desarrollo latinoamericano con la creación de los institutos de cáncer. Así:

- Venezuela, 1929. Instituto Luis Razetti.

- Colombia, 1934. Instituto Nacional de Radio.

- EE.UU., 1937. National Cancer Insitute (NCI).

- Brasil, 1938, Servicio de cancerología.

- Perú 1939. Instituto Nacional de cáncer.

- México 1946. Instituto de Cancerología.

Así mismo, relata la creación de las sociedades científicas de cancerología en el Perú y en el mundo, así: American Society of Cancer and Oncology (ASCO) en 1964, Federación Latinoamericana de sociedades de Cáncer (FLASCA) en 1963, European Society for Mecial Oncology (ESMO) en 1975 y SLACOM en 2003. En el Perú menciona la participación de la sociedad civil con la creación de la Liga Peruana Contra el Cáncer en 1950, estimulada por el Dr. Oscar Soto, y la instalación de la primera bomba de cobalto en 1958.
Posteriormente, la Srta. Frida Heller y Eduardo Cáceres crean la Fundación Peruana de Cáncer en 1960.

El desarrollo de la lucha contra el cáncer a nivel mundial continuó; así, como cuandoel presidente Richard Nixon declaró la guerra contra el cáncer, asignando 1500 millones de dólares. Después, con el liderazgo de Joe Biden, vicepresidente de EE.UU, cuyo hijo falleció de un tumor cerebral, el presidente Obama lanzó un moonshot con un presupuesto de 1,8 billones de dólares.

En el Perú, las acciones contra el cáncer continuaron y en el seguro social el Dr. Eduardo Valdivia Ponce creó el servicio de ginecología oncológica.

El INEN se mudó a su nuevo local en 1987 y se creó el voluntariado para permitir la operación del nuevo local.

En 1987 se creó PROSOMO, programa social que permitía el acceso a los cotosos medicamentos oncológicos a todos los pacientes y que incluía tanto al INEN como a la seguridad social, las fuerzas armadas y el sector privado. El programa fue muy exitoso y contó con el apoyo del presidente Alan García quien entrego un fondo semilla de un millón de dólares. Se atendió a muchos pacientes y el mismo fondo generó ingresos por 1,5 millones de dólares y un stock de medicinas por 300 mil dólares. Muchos pacientes pobres se beneficiaron pero, lamentablemente, por falta de visión o mezquindad política fue desactivado por el nuevo gobierno.

El INEN tomó nueva energía, en el año 2002, con un nuevo impulso a la investigación, se retomó el concepto de prevención, se creó el programa de descentralización y desconcentración, se inauguraron las unidades oncológicas en distintos hospitales de Lima y se desarrollaron los institutos regionales de enfermedades 
neoplásicas: en Trujillo el IREN Luis Pinillos Ganoza y en Arequipa el IREN Sur.

El claro concepto de cobertura nacional y estrategia común llegó a convocar a todos los actores en el tema cáncer, y juntos se creó un plan estratégico y luego el plan nacional para la prevención y control del cáncer con una estrategia a 10 años.

A través de la Coalición Nacional Multisectorial Perú contra el Cáncer se lanzó el plan con la presencia de todos los ex ministros de salud del Perú y con la presencia internacional del comité de ética multinacional de la Agencia Internacional de Investigación en Cáncer.

El INEN sigue desarrollándose, se hace progresos en el servicio de pediatría oncológica y el de medicina oncológica, llegando el momento de los trasplantes, e inaugurándose el servicio de trasplantes en el 2011.

El INEN desarrolló estudios en biología molecular, hizo los primeros trasplantes y logró convertirse en Organismo Público Descentralizado(OPD) en el 2006 y logró manejar su presupuesto por resultados en el 2011. Se desarrolló la iniciativa del Dr. Henry Gómez, el servicio de quimioterapia satelital, siendo directora del INEN la Dra. Tatiana Vidaurre.

El libro tiene también interesantes apuntes como la atención de la primera paciente, atendida el 1 de mayo 1940, de nombre Natalia A. L. Comenta el desarrollo de la cirugía progresivamente radical y se presenta el caso de una paciente sometida a hemicorporectomía por un sarcoma. El libro resalta hitos de la investigación en el país., como el del Dr. Pedro Weiss sobre el linfoma nasal o del Dr. Raúl León Barúa con su equipo en Helicobacter pylori y cáncer de estómago, y las múltiples investigaciones de los doctores Vallejos, Henry Gómez y otros; y, la introducción de la inmunoterapia.
También se refiere a la participación comunitaria y a la difusión por radio de Elmer Huerta, discípulo y amigo del Dr. Vallejos y de quien habla, y el programa radial del INEN con el Dr. Félix Bautista y la Dra. Gabriela Calderón.

Los avances nacionales en vacunación para el papilomavirus humano y hepatitis B. La importancia de la interacción con los gobiernos para la obtención de recursos sin los cuales los planes quedarían como tales, y con los legisladores para el apoyo político y aprobación de leyes.

Un interesante capitulo es el de los fraudes y mitos, y termina el libro con un listado cronológico que hubiera podido ser lo único que les presente.

Finaliza con un epilogo que nos trae la preocupación de que los casos de cáncer se duplicaron en 25 años y que si no actuamos se volverán a duplicar en los próximos 15 años.

Refiere los cuatro pilares del Dr. Peter Boyle en esta lucha que dice: Prevenir lo prevenible, tratar lo tratable, curar lo curable y paliar lo no curable.

Finaliza agradeciendo a su familia y amigos, pero creo que somos nosotros los que debemos agradecerle por lo hecho por todos nosotros en la investigación, mentoría, educación, manejo de sus pacientes y su liderazgo para el desarrollo de la oncología y la salud pública; personalmente, por su amistad y el honor de presentar este libro que estoy seguro médicos y no médicos leerán con gran interés,

\section{Palabras Del Autor, Dr. Carlos Vallejos Sologuren}

Buenas noches, queridos amigos. Quiero agradecerles por acompañarme hoy en la presentación de este libro. 
Hacer paralelos entre la historia y el progreso de una disciplina científica nos permite ver que todos compartimos un mismo camino que avanza inexorablemente. Sin embargo, lo que sí cambia es la velocidad con la que ciertos eventos transitan por ese sendero.

Desde los primero registros históricos del cáncer, hace más de tres mil años, en los papiros egipcios hasta el desarrollo de la capacidad de adaptar linfocitos para combatir al cáncer, creo que el camino para conocer esta enfermedad lo hemos venido recorriendo cada vez de manera más rápida, con una curva de aprendizaje en las últimas tres décadas mucho más acelerada que en toda la historia de la humanidad. El camino que me ha tocado compartir en este sendero me ha permitido ser testigo de muchos eventos, algunos de ellos fracasos de los cuales aprendimos; y, otros, triunfos inimaginables. Así, he aprendido no sólo a respetar al "enemigo" sino a admirarlo en su complejidad y como escribió Orson Scott Card: a amarlo para poder destruirlo.

Y, como he tenido el privilegio de presenciar en este andar los avances y, probablemente, la derrota final de este mal -al igual que de muchos otros- esta lucha se basó en tres pilares fundamentales, sin uno de los cuales las iniciativas colapsarían:

I. El conocimiento, sin el cual no existen fundamentos.

II. La voluntad, sin la cual el conocimiento simplemente se extravía.

III. La coyuntura, que son todos los factores que siendo anexos permiten que la teoría y la voluntad se plasmen en políticas de salud históricas que se traducen en hechos.
Afortunadamente, el conocimiento ha fluido desde y hacia todos los continentes, puesto que todos reconocemos en el cáncer a un enemigo común. Pero, en el Perú han vivido individuos históricamente únicos como el doctor Eduardo Cáceres Graziani o el doctor Andrés Solidoro Santisteban. Puedo decir con gratitud que fueron una parte crucial de mi desarrollo como médico oncólogo. Gracias a su apoyo tuve la oportunidad de ser admitido en el MD Anderson, el mejor hospital en el mundo para el manejo del cáncer, y luego regresar a trabajar en el INEN, el mejor hospital oncológico de la región.

En una ocasión como esta, no puedo dejar de recordar con un cariño muy especial y brindar tributo al Dr. Emil J Freireich, al Dr. Emil Frei y al Dr. Robert L. Comis, sin cuyos valiosos ejemplos, enseñanzas y guías, estoy seguro, mi carrera hubiera sido muy diferente.

Mi aprecio también a quienes me dieron la oportunidad de poner un grano de arena en el desarrollo de la historia de esta lucha en nuestro país, experiencias que siendo un desafío enorme fueron sumamente enriquecedoras en lo profesional y en lo personal.

Es importante saber que aún existen instituciones en el Perú, como la Universidad Peruana Cayetano Heredia, que continúan apoyando iniciativas como la publicación de este libro. De la misma manera, quiero agradecer a todas aquellas personas que a través de sus sugerencias, críticas y aportes, hicieron de esta ambiciosa idea una magnifica realidad.

Espero que estas páginas, sencillas, pero resultado de un gran esfuerzo, sean un aporte para que nuevas generaciones de científicos se encuentren con la maravillosa complejidad del cáncer y los estimule a sumarse en el camino de su derrota.

Muchas gracias 